\title{
Bone in CKD: why the ERA EDTA CKD-MBD working group organized a dedicated meeting?
}

\author{
Sandro Mazzaferro ${ }^{1} \cdot$ Ziad A. Massy $^{2} \cdot$ Marc Vervloet $^{3} \cdot$ Mario Cozzolino $^{4}$
}

Published online: 23 September 2017

(C) Italian Society of Nephrology 2017

In recent years, we have witnessed an impressive increment of our knowledge of bone. Osteocytes, formerly regarded as inactive cells evolved from osteoblasts, victim of their own mineralizing activity, are now considered to be the master orchestrators of osteoblasts and osteoclasts and as such the core of bone turnover. In fact, osteocytes are not inactive or dead, rather they actively live although buried into the lacuno-canalicular system they created themselves. Fluid movements and forces in the lacunae allow bone to sense mechanical load and to react by producing local chemical signals that elicit, for example, the processes of microcracks repair. Eventually, osteocytes also produce (or modulates the production by osteoblasts and osteoclasts) a number of noncollagenous bone proteins that reach blood flow and activate specific receptors in distant organs, thus transforming bone into a systemic endocrine gland with potential distant effects. For this reason, the "mineral bone disorders" (MBD) that affect "chronic kidney disease" (CKD) patients (namely, CKD-MBD), encompasses more endocrine systems, besides the traditional roles of parathyroid hormone and vitamin D,

Sandro Mazzaferro

sandro.mazzaferro@uniroma1.it

1 Department of Cardiovascular, Respiratory, Nephrologic, Anaesthetic and Geriatric Sciences, Sapienza University of Rome, Rome, Italy

2 Division of Nephrology, Ambroise Paré University Hospital, APHP, Boulogne-Billancourt/Paris, and INSERM U1018, Team 5, CESP, UVSQ, Villejuif, France

3 Department of Nephrology and Cardiovascular Sciences (ACS), VU University Medical Center, Amsterdam, The Netherlands

4 Renal Division, Department of Health Sciences, University of Milan, ASST Santi Paolo e Carlo, San Paolo Hospital, Milan, Italy and now include some new players whose biologic role is even still incompletely understood, but clearly have clinical relevance. For example, the originally described mode of action of FGF23 in mineral homeostasis allowed to evidentiate part of the complex link between bone and kidney [1] and to better classify some metabolic diseases [2]. However, it is becoming increasingly evident that in chronic renal failure in particular, FGF23 (alone or in combination with other bone proteins) also explains in part the link between CKD and cardiovascular disease [3]. Further in renal insufficiency, bone strength is significantly impaired and leading to increased fracture rate and an impressive additional burden of morbidity and mortality [4]. Thus, the increased knowledge in bone physiology allows to explain how bone disease in renal failure can be part of the systemic manifestations of uraemia. For all these reasons, the ERA-EDTA working group on CKD-MBD sensed the importance of dedicating efforts to increase the awareness and clinical appreciation specifically of bone disease in renal patients. The dedicated symposium "bone in chronic kidney disease", organized in Amsterdam on 23 September 2016, involved internationally acknowledged experts in the field, including non-nephrologists, with the aim to highlight the emerging clinical aspects of renal osteodystrophy (ROD), and to provide tools to implement this knowledge into practice. The presentations of the meeting have been translated into papers that are now included in the present Special Issue of Journal of Nephrology. The subject of the relationship between uremia and bone beyond secondary hyperparathyroidism is examined in the paper by Fukagawa et al. that underlines how some uremic toxins can have direct negative effects on bone cells. The need to perform more bone biopsies now that we have increased our knowledge of bone cells, but importantly also our therapeutic armamentarium, has been evidenced in recent years [5]. In this respect, another 
relevant problem, examined in the paper by Evenepoel et al., is how to revitalize the procedure of bone biopsy, an expertise that has been lost almost completely by nephrologists in recent years, with the consequence that clinicians (including pathologists) with the required skills and experience are disappearing. A further interesting aspect of ROD is the impressive increment of the prevalence of adynamic bone disease, possibly secondary to our therapeutic efforts that, we are now discovering, are not always based on adequate knowledge of the underlying pathophysiology. According to the paper by Massy et al., adynamic bone could be present already in the very early stages of renal failure as a result of the initial adaptive response of bone, aiming at facing the early derangements of mineral metabolism in CKD. It is not known how exactly, but an increased expression of sclerostin in bone in early CKD could be responsible of low TO, with or without contribution from uremic toxins. If confirmed, this hypothesis would indicate that adynamic bone is not (or is not necessarily) the result of our inadequate therapies, rather, it would indicate that we need to know when and how this adaptive response becomes maladaptive [6]. This increased importance of early recognition and identification of bone disease rises the need to perform bone biopsies as outlined above, but also prompts renewed efforts for noninvasive identification of ROD types and of fracture risk by means of old and/or new radiologic techniques, as examined in the papers by Jannot et al. and by Urena-Torres et al. In addition to bone histology and radiological techniques less invasive biochemical assays may assist in diagnosing bone disease in CKD. For this reason, circulating bone biomarkers remain of relevance for both diagnostic purposes and to evaluate the efficacy of available therapies, as discussed in the paper by Vervloet et al. The last topic during the symposium consisted of therapeutic possibilities for renal osteodystrophy. The mainstay of bone disease has always been and still is vitamin $\mathrm{D}$ in the native or active/synthetic forms. The point of native vitamin $\mathrm{D}$ therapy is examined in general for osteoporosis patients without renal failure and with CKD (Lips et al.). However, in more advanced stages of renal insufficiency, the use of vitamin D, especially in the active/ synthetic formulations, is already diffuse but with rather limited clinical satisfaction, given the recently appreciated clinical burden of fractures in renal insufficiency. Therefore, the use of second-line drugs like bisphosphonates and other available therapies for osteoporosis is considered by Bover et al. (classic drugs for osteoporosis) and by Tartaglione et al. (new biologicals) in the last two papers. Indeed, in the field of osteoporosis, new biological drugs have been introduced, with apparently significant clinical results. These drugs directly modify the activity of the individual bone cell types, allowing driving the balance between bone formation and resorption. Theoretically, there could be room for administration of specific drugs in each specific subtype of ROD identified by bone histology. However, the presence of CKD carries potential drawbacks that the nephrologists need to be aware of in order to obtain the best benefit/harm ratio from these drugs. Importantly, the potential systemic, extraskeletal effects of these new therapies should be taken into consideration, as highlighted in the last paper in this issue. In summary, this special issue of the Journal of Nephrology provides a useful reference for both practical choices in the individual patient and theoretical considerations for research purposes, and is characterized by the best of our updated knowledges in the field of bone in CKD which are prepared by internationally appreciated experts under the umbrella of ERA EDTA CKD-MBD Working group.

\section{Compliance with ethical standards}

Conflict of interest The authors declare that they have no competing interests.

Ethical approval This article does not contain any studies with human participants performed by any of the authors.

\section{References}

1. Wei K, Yin Z, Xie Y (2016) Roles of the kidney in the formation, remodeling and repair of bone. J Nephrol 29:349-357

2. Mazzaferro S, Pasquali M, Pirrò G, Rotondi S, Tartaglione L (2010) The bone and the kidney. Arch Biochem Biophys 503(1):95-102. doi:10.1016/j.abb.2010.06.028 (Epub 2010 Jul 3)

3. Vervloet MG, Massy ZA, Brandenburg VM, Mazzaferro S, Cozzolino M, Ureña-Torres P, Bover J, Goldsmith D, CKD-MBD Working Group of ERA-EDTA (2014) Bone: a new endocrine organ at the heart of chronic kidney disease and mineral and bone disorders. Lancet Diabetes Endocrinol 2(5):427-436. doi:10.1016/ S2213-8587(14)70059-2

4. Tentori F, McCullough K, Kilpatrick RD, Bradbury BD, Robinson BM, Kerr PG, Pisoni RL (2014) High rates of death and hospitalization follow bone fracture among hemodialysis patients. Kidney Int 85(1):166-173. doi:10.1038/ki.2013.279

5. Carvalho C, Moniz Alves C, Frazão JM (2016) The role of bone biopsy for the diagnosis of renal osteodystrophy: a short overview and future perspectives. J Nephrol 29:617-626

6. Wolf M (2015) Mineral (Mal) adaptation to kidney diseaseYoung investigator award address: American society of nephrology kidney week 2014. Clin J Am Soc Nephrol 10(10):1875-1885 